\title{
EXPERIMENTAL INFECTION OF THE NEOTROPICAL MALARIA VECTOR ANOPHELES DARLINGI BY HUMAN PATIENT-DERIVED PLASMODIUM VIVAX IN THE PERUVIAN AMAZON
}

\author{
AJAY R. BHARTI, RAUL CHUQUIYAURI, KIMBERLY C. BROUWER, JEFFREY STANCIL, JESSICA LIN, \\ ALEJANDRO LLANOS-CUENTAS, AND JOSEPH M. VINETZ* \\ University of California San Diego School of Medicine, La Jolla, California; Instituto de Medicina Tropical Alexander von \\ Humboldt, Universidad Peruana Cayetano Heredia, Lima, Peru; Naval Medical Research Center Detachment, Iquitos, Peru
}

\begin{abstract}
Malaria transmission from humans to mosquitoes is modulated by human host immune factors. Understanding mechanisms by which the human host response may impair parasite infectivity for mosquitoes has direct implications for the development of transmission-blocking vaccines. We hypothesized that despite a low transmission intensity of malaria in the Peruvian Amazon region of Iquitos, transmission-blocking immunity against Plasmodium vivax might be common, given an unexpectedly high proportion of asymptomatic parasitemic individuals in this region. To test this hypothesis, the ability of symptomatic $P$. vivax malaria patients to experimentally infect wild-caught outbred Anopheles darlingi mosquitoes was tested using the indirect membrane feeding technique. Only half (52/102) of $P$. vivax parasitemic patients successfully infected mosquitoes. Transmitters were more likely to have gametocytes $(\mathrm{OR} 6.35$, $P=0.003$ ), high parasitemia (OR 3.79, $P=0.024)$, and, in terms of basic clinical parameters, a slower pulse rate (mean \pm SD: $82.3 \pm 12.3$ versus $88.7 \pm 13.5, P=0.016)$ than non-transmitters. $\log _{10}$ gametocytemia and $\log _{10}$ real-time reverse transcriptase Pvs25 PCR quantifying gametocytes were significantly and positively correlated with oocyst counts (correlation coefficient $0.505, R^{2}=0.26, P=0.001$ ). These experiments are the first to establish a system of determining transmission patterns in experimental infection of outbred natural neotropical malaria vectors in the Amazon region. Patients with $P$. vivax inefficiently infect outbred $A n$. darlingi mosquitoes, raising the possibility that some degree of naturally occurring transmission-blocking immunity is present on a population basis in the Peruvian Amazon, an area of low intensity of malaria transmission.
\end{abstract}

\section{INTRODUCTION}

Malaria transmission begins with uptake of viable, mature gametocytes by female Anopheles mosquitoes during ingestion of an infectious blood meal. Patients harboring malaria parasites are not equally infectious to mosquitoes. Naturally occurring transmission-blocking and transmission-enhancing activity (both immune and non-immune mediated) present in serum has been demonstrated both for Plasmodium falciparum and Plasmodium vivax in endemic regions. Nonetheless, molecular targets of $P$. vivax transmission-blocking immunity have not been delineated in the field setting. ${ }^{1-3}$

Infectivity of Plasmodium gametocytes for mosquitoes has been shown to be modulated by antibody, ${ }^{4}$ complement,${ }^{5}$ and leukocytes. ${ }^{6}$ This combination of factors leads to variability in the ability of gametocytemic individuals to infect mosquitoes. Intuitively, the three major determinants would be parasite infectivity, vector competence, and host factors (genetic, immune, and non-immune). Quantity of gametocytes as determined by light microscopy has not been demonstrated to predict $P$. vivax transmission to mosquitoes. ${ }^{7}$

$P$. vivax is more common than $P$. falciparum as a cause of malaria in many parts of the tropics and subtropics outside of sub-Saharan Africa. ${ }^{8}$ Malaria caused by $P$. vivax occurs in many regions of Asia and Latin America but has been understudied and underappreciated as a public health threat there. ${ }^{8}$ In Peru, the incidence of malaria has increased dramatically since the early 1990 s. $^{9} P$. vivax remains the pre-

\footnotetext{
* Address correspondence to Joseph M. Vinetz, University of California San Diego School of Medicine, Division of Infectious Diseases, Department of Medicine, 9500 Gilman Drive, 0741, George Palade Laboratories/Cellular and Molecular Medicine-West Room 125, La Jolla, CA 92093-0741. E-mail: jvinetz@ucsd.edu
}

dominant cause of malaria in the region and is a considerable source of morbidity to people of all ages, especially during pregnancy where it is associated with maternal anemia and low birthweight. ${ }^{10}$

The principal malaria vector in the Amazon basin is Anopheles darlingi, an aggressive, anthropophilic mosquito that has invaded the western Amazon over the past 15 years due, at least in part, to deforestation, ${ }^{11}$ and has led to epidemic malaria in the region. ${ }^{12}$ Despite being outnumbered by another malaria-competent anopheline mosquito, Anopheles benarrochi (71\% vs. $24 \%$ ), An. darlingi has been found to have a much higher infection rate ( $1 \%$ vs. $0.14 \%)$, which, combined with being highly anthropophilic, has led to its being the most important malaria vector in the Amazon basin. ${ }^{13,14}$ However, because An. darlingi cannot be propagated in colonies, the natural $P$. vivax-An. darlingi combination has been difficult to study under experimental settings using infected humans as sources of infectious blood meals to determine the biologic parameters of transmission. To do so requires a logistically challenging system in which $A n$. darlingi is caught in the field, induced to lay eggs to obtain uninfected F1 mosquitoes, and infected with blood from a naturally infected human in the laboratory setting. Only in this experimental setting can naturally acquired immunity that closely mimics the field setting be simulated.

In areas of high malaria transmission, patients develop clinical immunity as a result of an immune response that controls, but does not eliminate, parasitemia while simultaneously avoiding severe pathology. This phenomenon is well described for $P$. falciparum $^{15}$ and more recently for $P$. vivax ${ }^{16-18}$ malaria. Interestingly, in the Peruvian Amazon, a site of low malaria transmission intensity, we and others have demonstrated an unexpectedly high prevalence of asymptomatic malaria with one-third of slide-positive and three-fourths of PCR-positive patients being asymptomatic. ${ }^{19,20}$ These pa- 
tients serve as a reservoir of infection, but the transmission pattern in this group is unknown.

This study was conducted to determine the ability of $P$. vivax-infected patients in the Peruvian Amazon to infect $A n$. darlingi mosquitoes. We hypothesized that, despite low endemicity, transmission-blocking immunity develops against $P$. vivax in the Peruvian Amazon. Using the natural outbred $A n$. darlingi-P. vivax pairing, we also determined the relationship between $P$. vivax parasitemia, gametocytemia as determined by light microscopy and quantitative reverse transcriptase PCR, and clinical features and infectivity for $A n$. darlingi mosquitoes.

\section{MATERIALS AND METHODS}

Study site. Patient enrollment and experimental mosquito infections were carried out in Iquitos, the capital city of the department of Loreto, Peru, over a period of 14 months from May 2004 to June 2005. Enrollment criteria were patients with acute fever and $P$. vivax parasitemia. The region comprises nearly one-fourth of the landmass of Peru and has the ecological characteristics of the Amazon lowlands. Malaria cases occur nearly throughout the year, with seasonal peaks from January to September. $P$. vivax prevalence in this population is 5 times more than that of $P$. falciparum $(14 \%$ vs. $3 \%)$ as has been shown by active surveillance. ${ }^{19}$

Patient recruitment method and data collection. This study was approved by the Institutional Review Boards/Ethical Committees of the University of California San Diego, Universidad Peruana Cayetano Heredia, A.B. Prisma, and United States Department of Defense. Permission to carry out the study was provided by the Loreto Director of Health, Iquitos, Peru. Informed consent was obtained from all participants prior to enrollment. All participation was voluntary, and subjects could opt out at any time during the study. All parasitemic patients, regardless of decision to participate, were offered free anti-malarial chemotherapy according to Peruvian Ministry of Health guidelines. Patients were sought from the outpatient department of Hospital Apoyo Iquitos and the health posts at the villages of Varillal and Santo Tomas. No patient had been on anti-malarial therapy on entry to the study. Febrile patients (defined as having an oral temperature $>37.7^{\circ} \mathrm{C}$ ) routinely have blood smears made when presenting for care. Samples were prepared for thick and thin smear microscopy using 5\% Giemsa stain, and the slides were examined on site by an experienced Ministry of Health microscopist. This diagnosis was considered standard for inclusion of patients in the study. Patients were excluded if they had severe or complicated malaria as defined by the World Health Organization, serious co-morbid conditions (e.g., HIV/AIDS or malnutrition; none were excluded for these reasons), or were pregnant ( 2 were excluded). Symptomatic $P$. vivax patients 18 years and older were invited to participate in the study, and informed consent was obtained from those willing. Data regarding age, sex, occupation, place of residence, and history of travel were obtained. Physical examination was done and included general appearance, heart rate, respiratory rate, pallor, icterus, and splenomegaly. Information regarding past history of malaria (type, frequency), date of onset of current symptoms, and use of drugs (anti-malarials and anti-pyretics) was noted.
Mosquitoes. An. darlingi mosquitoes were caught at night using human bait in the study villages under U.S. Department of Defense-approved protocols, including strict attention to occupational safety, provision of malaria prophylaxis, and surveillance for malaria as the cause of febrile illness. Adult female An. darlingi mosquitoes were brought to the Iquitos insectary and fed on chicken blood to induce egg-laying, and F1 mosquitoes were then reared to adults using established methods. ${ }^{21}$ Mosquitoes were fed on parasite-containing blood 3-4 days post-emergence.

Membrane-feeding assays. A member of the study team accompanied the patients to the insectary, and a physician was present throughout the procedure. Blood samples were obtained in heparinized tubes, maintained at $37^{\circ} \mathrm{C}$, and blood smears repeated. Two and a half milliliters of blood was added to a 5-cm diameter hand-blown glass feeder (Perpetual Systems Corp., Rockville, MD) closed with Parafilm (American National Can, Chicago, IL). To prevent exflagellation of microgametocytes, a constant temperature of $37^{\circ} \mathrm{C}$ was maintained using a water-jacketed circulation system. ${ }^{22}$ The mosquitoes (25 per carton) were allowed to feed for $15 \mathrm{~min}$ in the dark, after which time the glass membrane feeder was removed from the top of the carton and unengorged mosquitoes were removed. Blood samples from each patient were fed to mosquitoes in 3 separate cartons simultaneously. After feeding, all mosquitoes were kept in the insectary $\left(22-27^{\circ} \mathrm{C}\right.$ and $70-80 \%$ humidity) and provided with $20 \%$ sucrose daily until they were dissected on days 7, 8, and 9 after feeding. Midguts were dissected in a drop of phosphate-buffered saline, and oocysts counted by examination under $10 \times$ light microscopy. Immediately after blood draw, patients were given antimalarial drugs (chloroquine and primaquine) per Peruvian Ministry of Health guidelines. A second blood smear was prepared at the time of blood draw to confirm the diagnosis and to quantify parasitemia and gametocytemia. The following system was used: $<1+$, at least 1 parasite/100 high-power field (HPF); 1+, 1 parasite/HPF; 2+, 2-20 parasites/HPF; 3+, 21-200 parasites/HPF; 4+, > 200 parasites/HPF.

Real-time reverse transcriptase PCR. A real-time reverse transcriptase PCR based on the Pvs 25 gene (GenBank accession no. AB033343) was developed as an alternative method for quantifying $P$. vivax gametocytemia. ${ }^{23}$ Primers (forward, 5'-AACGAAGGGCTGGTGCACCTTT-3', 5' -AGCAACCTGCACTTTGGATTTCCG-3') were designed to amplify $P v s 25$. The predicted fragment size of $267 \mathrm{bp}$ was amplified from total genomic DNA obtained from whole blood containing $P$. vivax from a patient. The amplified product was cloned into the pCR 2.1-TOPO plasmid vector. A tenfold dilution of the plasmid DNA starting with $10^{10}$ plasmid copies $\mu \mathrm{L}$ (as determined by spectrophotometry at $\mathrm{OD}_{280}$ ) was used as a positive control and for making a standard curve. RNA isolation from freshly obtained infected blood from Peru was done using the PAXgene Blood RNA Kit (Qiagen Inc., Valencia, CA). Briefly, $2.5 \mathrm{~mL}$ of whole blood was collected in PAXgene Blood RNA Tubes (Becton Dickinson (BD), Franklin Lakes, NJ, catalog no. 76216) and stored at $-20^{\circ} \mathrm{C}$ after being allowed to incubate at room temperature for 2 hours. All samples were processed within 2 weeks of collection using the PAXgene Blood RNA Kit. TaqMan probes were designed for the Pvs 25 real-time PCR assays. A 5' 6-FAM reporter dye was selected, with a 3' Black Hole Quencher on the end. Probe concentration was optimized at 
a final concentration of $0.1 \mu \mathrm{M}$ in each reaction, primer final concentration was $0.2 \mu \mathrm{M}$, and $10 \mu \mathrm{L}$ of RNA template from each extraction was used for each reverse transcriptase PCR reaction. The reverse transcriptase PCR cycles were as follows: $50^{\circ} \mathrm{C} \times 15$ minutes, $95^{\circ} \mathrm{C} \times 2$ minutes, $94^{\circ} \mathrm{C} \times 10$ seconds, $55^{\circ} \mathrm{C} \times 45$ seconds, plate read, 45 cycles total. For each reverse transcriptase PCR run, RNA samples were run alongside tenfold dilutions of the plasmid DNA that were performed in triplicate to generate a reliable standard curve. For each RNA sample subjected to reverse transcriptase PCR (using Invitrogen's one-step reverse transcriptase PCR reagents with reverse transcriptase and Taq polymerase), a reverse transcriptase minus control was run using the standard PCR reagents with Taq polymerase only for contaminating genomic DNA. The real-time probe used was the 5' 6-FAMAACCCAGACCCAGCACAGGTAAACATG-3' Black Hole Quencher.

Statistical analysis. For analysis purposes, a person was defined as a "transmitter" if a blood sample from that person had successfully infected at least one mosquito. Univariate analyses, using the $\chi^{2}$ test or $t$ test to compare means where appropriate, were used to examine which variables were associated with transmission of infection to mosquitoes. A $t$ test was also used to compare the mean percentage of mosquitoes infected by parasitemia group, and the Mann-Whitney $U$ test was used to compare the number of oocysts per infected midgut with parasitemia group. Relationships between real-time reverse transcriptase PCR quantification of gametocytes, gametocytemia, oocysts per infected midgut, and percentage of mosquitoes infected were compared using simple linear regression. Two-sided $P$ values $<0.05$ were considered statistically significant. Data were analyzed using the SPSS 12.0 (SPSS, Inc., Chicago, IL) statistical package.

\section{RESULTS}

Patient data. A total of 102 patients with symptomatic $P$. vivax infection were enrolled in the study (Table 1). The sex ratio was approximately 2:1 with 65 (64\%) men and $37(36 \%)$ women. The mean age was $34 \pm 12$ SD years (range 18-63 years). All had at least one of the following clinical symptoms by history: fever, chills, or headache (except for 1 patient for whom data were missing). Most patients (80\%) had all 3 symptoms on presentation and were seen within 7 days from onset of illness. Sixty percent (60/102) of the patients gave history of prior malaria episodes. About two-thirds (69/102) of patients admitted to taking an analgesic, anti-pyretic, or anti-inflammatory medicine (acetaminophen, ibuprofen, naproxen, or aspirin) some time during the course of their illness. At the time of membrane feeding, half (51/102) of the patients were febrile, with a temperature of $37.5^{\circ} \mathrm{C}$ or more (range $37.7-40.1^{\circ} \mathrm{C}$ ). Pulse rate ranged from 60 to 120 beats per minute with a mean of $85.4 \pm 13.2$ SD. Pallor was noted in $65 \%(66 / 102)$ of patients, but because the measurements were not standardized, this variable was not included in the analysis.

Results of light microscopy quantification of $P$. vivax parasitemia. Diagnoses of all patients reported as having $P$. vivax malaria by Ministry of Health microscopists at all study sites were confirmed independently in our laboratory. The smear results ranged from $<1+$ to $3+$, with a majority of them falling in the $2+$ group (77/102). Gametocytemia ranged from 0 to 106 gametocytes per $100 \mathrm{HPF}$ with a mean of 20.61 .
TABLE 1

Comparison of clinical aspects of patients who transmitted vs. those that did not transmit $P$. vivax infection to mosquitoes*

\begin{tabular}{|c|c|c|c|}
\hline & $\begin{array}{c}\% \text { Patients that } \\
\text { infected } \\
\text { mosquitoes } \\
(n=52)\end{array}$ & $\begin{array}{l}\text { \% Patients that } \\
\text { did not infect } \\
\text { mosquitoes } \\
(n=50)\end{array}$ & $P$ value \\
\hline \multicolumn{4}{|l|}{ Sex } \\
\hline$\%$ male & 64 & 64 & 0.96 \\
\hline \multicolumn{4}{|l|}{ Age (years) } \\
\hline$<25$ & 31 & 35 & 0.35 \\
\hline $25-34$ & 31 & 18 & \\
\hline$\geq 35+$ & 39 & 47 & \\
\hline Mean \pm SD & $32.6 \pm 10.9$ & $34.8 \pm 13.6$ & 0.37 \\
\hline \multicolumn{4}{|l|}{ Past malaria episodes (a) } \\
\hline Had malaria in past & 67 & 66 & 0.94 \\
\hline \multicolumn{4}{|l|}{ Chills } \\
\hline Present & 96 & 96 & 0.97 \\
\hline \multicolumn{4}{|l|}{ Headache } \\
\hline Present & 100 & 98 & 0.31 \\
\hline \multicolumn{4}{|l|}{ Fever } \\
\hline$\geq 37.5^{\circ} \mathrm{C}$ & 44 & 56 & 0.24 \\
\hline$\geq 40.0^{\circ} \mathrm{C}$ & 6 & 12 & 0.31 \\
\hline \multicolumn{4}{|l|}{ Fever medications } \\
\hline Recent history of use & 73 & 72 & 0.90 \\
\hline \multicolumn{4}{|l|}{ Pulse (b) } \\
\hline Mean \pm SD & $82.3 \pm 12.3$ & $88.7 \pm 13.5$ & 0.016 \\
\hline \multicolumn{4}{|l|}{ Parasitemia (categories) } \\
\hline Groups 1-2 & 8 & 24 & 0.024 \\
\hline Groups 3-4 & 92 & 76 & \\
\hline \multicolumn{4}{|l|}{$\begin{array}{l}\text { Gametocytemia by smear } \\
\text { (categories) }\end{array}$} \\
\hline 0 vs. any $(>0)$ & 94 & 72 & 0.003 \\
\hline
\end{tabular}

* Boldface indicates statistically significant association.

(a) $n=101$ for past malaria episodes; (b) $n=98$ for pulse.

Infectivity of $\boldsymbol{P}$. vivax patients for An. darlingi mosquitoes. A total of 4,017 mosquitoes successfully fed on infected blood using the artificial membrane feeding technique, with 2,631 $(65 \%)$ surviving until the time of midgut dissection. Approximately $50 \%(52 / 102)$ of the patients specimens infected mosquitoes, and $23 \%(602 / 2,631)$ of mosquitoes had at least one reported oocyst. The mean oocyst load per infected midgut was $11.5 \pm 25.9$ with a range of $1-175$.

Relationship of $P$. vivax parasitemia and gametocytemia to transmission of infection to mosquitoes. In $P$. vivax transmitters, the frequency of high parasitemia $(2+$ or $3+)$ was higher than in non-transmitters ( $92 \%$ vs. $76 \%)$. The odds of $P$. vivax transmission to mosquitoes for those with high $(2+$ or $3+)$ versus low parasitemia $(<1+$ or $1+)$ were statistically significantly greater (Odds Ratio 3.79, 95\% CI: 1.13-12.7, $P=$ 0.024). The higher parasitemia groups also had a greater mean percentage of mosquitoes infected (Figure 1a, $P=$ 0.001 by $t$ test) and a borderline statistically significant greater number of oocysts per infected midgut (Figure $1 \mathrm{~b}, P=0.09$ by Mann-Whitney $U$ test). After grouping the cases into those with or without gametocytes on smear examination, the odds of $P$. vivax transmission for those with gametocytes versus no gametocytes were significantly higher (OR 6.35, 95\% CI: 1.70-23.8, $P=0.003)$.

Results of real-time reverse transcriptase PCR. Real-time PCR data were available for 37 patients; linear regression showed a significant and positive correlation between $\log _{10}$ gametocytemia and $\log _{10}$ real-time reverse transcriptase PCR results $\left(R^{2}=0.26, P=0.001\right.$; Figure 2$)$; $\log _{10}$ gametocytemia and the percentage of mosquitoes infected $\left(R^{2}=\right.$ $0.23, P<0.001$; Figure 3 ); and $\log _{10}$ gametocytemia and $\log _{10}$ 
a

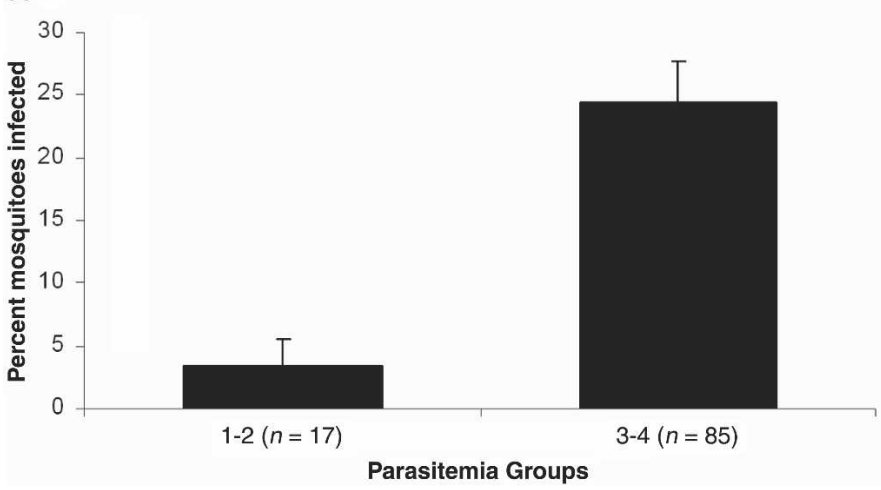

b

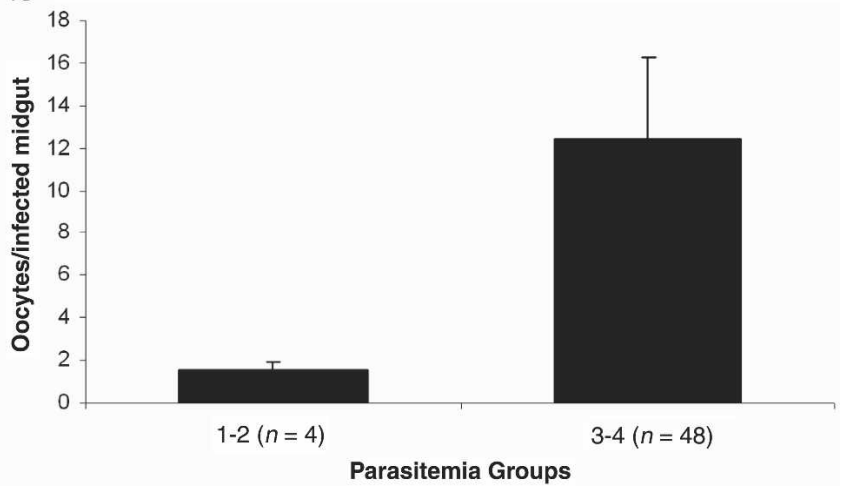

FIGURE 1. Relationship between $P$. vivax parasitemia and (a) percent of $A n$. darlingi mosquitoes infected $(P=0.001$ by $t$ test) or $(\mathbf{b})$ oocyst density in infected midguts $(P=0.09$ by Mann-Whitney $U$ test $)$. Groups $<1+$ and $1+$ vs. $2+$ and $3+$ include patients with $<2$ and $\geq 2$ parasites per HPF, respectively, on peripheral blood smears. Error bars indicate standard error of mean.

oocysts/midgut $\left(R^{2}=0.26, P=0.001\right.$; Figure 4$)$. The correlation between $\log _{10}$ real-time reverse transcriptase PCR and $\log _{10}$ oocysts/midgut was not statistically significant, but that between $\log _{10}$ real-time reverse transcriptase PCR and percentage of mosquitoes infected was statistically significant $\left(R^{2}=0.21, P=0.004\right.$; Figure 5). Univariate analysis of pulse using Student's $t$ test to compare means shows that transmitters had a lower mean pulse than non-transmitters (mean \pm standard deviation: $82.3 \pm 12.3$ vs. $88.7 \pm 13.5$, respectively; $P=0.016)$. There was no clear relationship between fever $\left(\geq 37.5^{\circ} \mathrm{C}\right)$ at time of membrane feed and transmission ( $44 \%$ vs. $56 \%$ for transmitters versus non-transmitters, respectively).

\section{DISCUSSION}

Four major findings emerged from this study. First, we successfully infected an outbred group of An. darlingi mosqui-

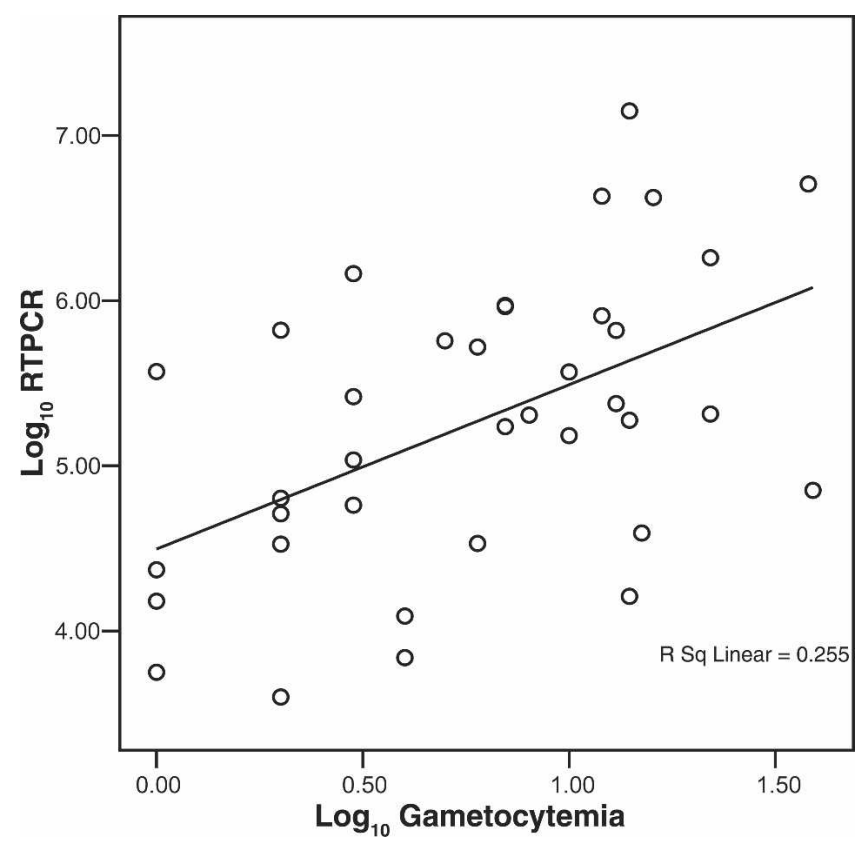

Figure 2. Association of $\log _{10} P$. vivax gametocytemia (excluding null values) and $\log _{10}$ real-time reverse transcriptase PCR quantification of $P v s 25$ mRNA; $R^{2}=0.26, P=0.001, N=37$. toes with $P$. vivax obtained ex vivo from malaria patients. Second, there was incomplete and inefficient transmission of infection from patients (half of $P$. vivax parasitemic patients) to outbred $A n$. darlingi mosquitoes in an area with low malaria endemicity. Third, $P$. vivax transmitters were more likely to have gametocytes on smear, high parasitemia, and a lower mean pulse than non-transmitters. Fourth, there was a correlation between smear and real-time reverse transcriptase PCR gametocytemia.

Our findings are in agreement with other published data where symptomatic $P$. vivax patients were studied. In studies where mosquitoes were allowed to feed directly on symptomatic Thai $P$. vivax patients, there was successful transmission of infection from $63 \%$ to $73 \%$ cases with a mean oocyst load of $9-13.4$ and a mean frequency of infection of $43-57 \%$ of mosquitoes fed. ${ }^{7,24}$ Similarly, membrane feeds using blood from $P$. vivax-infected symptomatic Thai patients resulted in

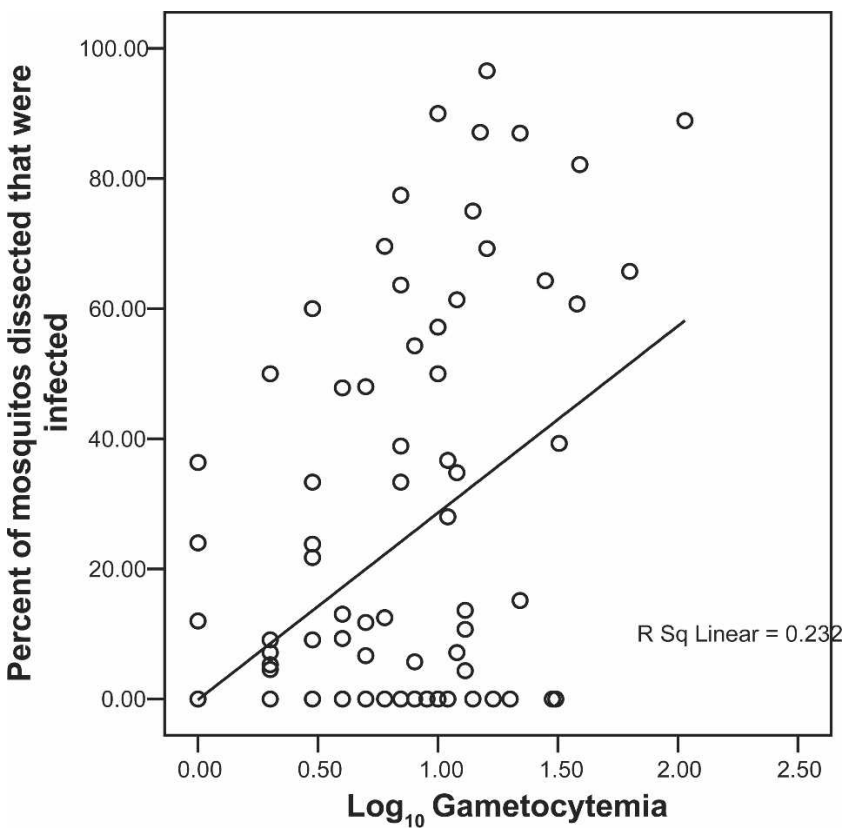

Figure 3. Association of $\log _{10}$ gametocytemia and percent of $A n$. darlingi mosquitoes infected by membrane feeding assay with exvivo, human-derived $P$. vivax; $R^{2}=0.23, P<0.001, N=98$. 


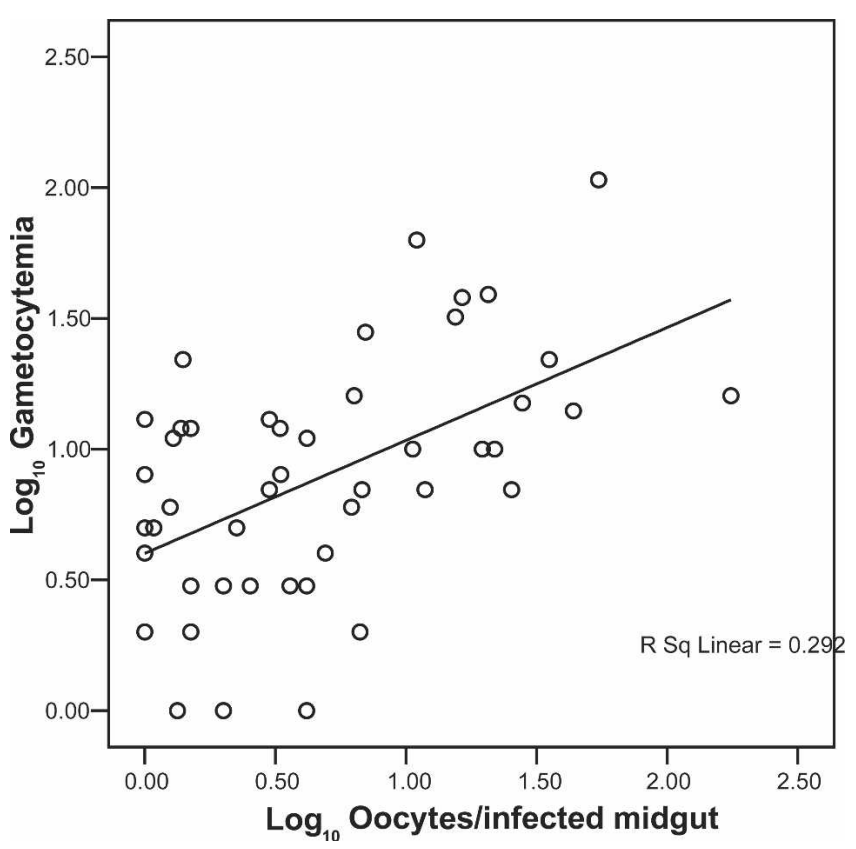

FIGURE 4. Association of $\log _{10}$ gametocytemia and $\log _{10}$ oocysts per midgut of $A n$. darlingi infected by membrane feeding assay with ex-vivo, human-derived $P$. vivax $; R^{2}=0.29, P<0.001, N=50$.

a mean oocyst load of 8.8 with $50.3 \%$ mosquitoes infected. ${ }^{24}$ One important difference in these studies was that the mosquitoes used were Anopheles dirus; these have been raised in colonies for over 25 years and are genetically inbred. Our study confirms a previous, smaller study in which wild-caught An. darlingi mosquitoes were infected in the Brazilian Amazon. ${ }^{25} \mathrm{We}$, too, demonstrate that experimental infection of a non-colonizable Anopheles spp. mosquito is feasible. The

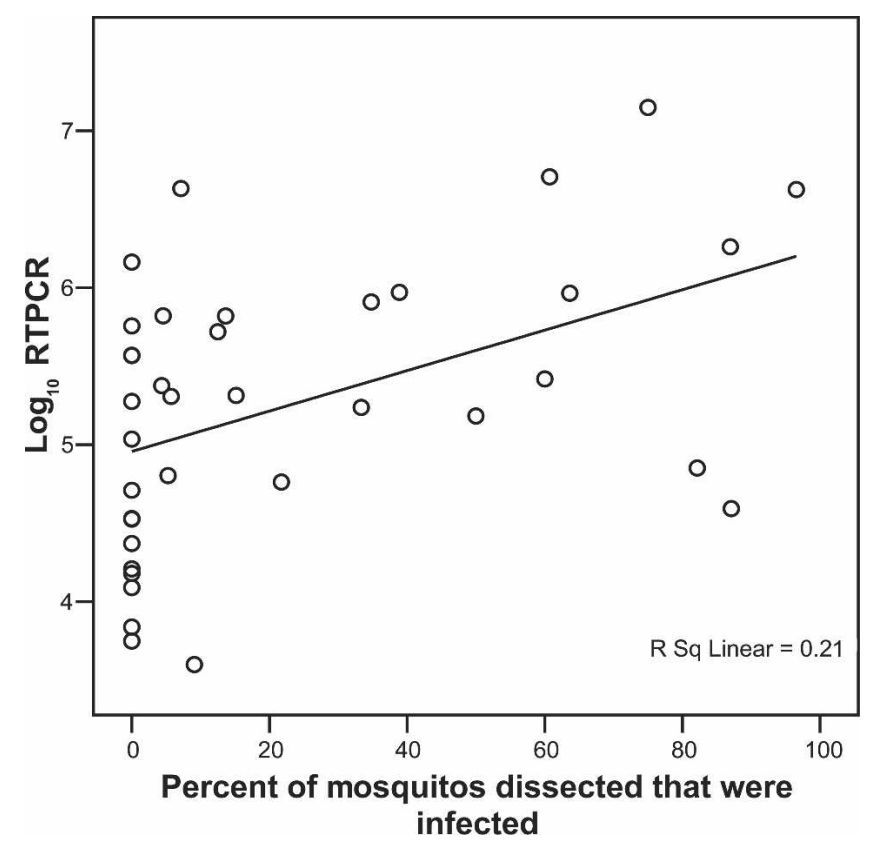

FIGURE 5. Association of $\log _{10}$ real-time PCR-determined Pvs 25 copy number and proportion of An. darlingi that were successfully infected with at least one oocyst; $R^{2}=0.21, P=0.004$. present study, however, provides a basis for applying such a system to assess mechanisms of transmission-blocking immunity in the endemic field setting.

Naturally occurring transmission-blocking immunity has been demonstrated both for $P$. falciparum and $P$. vivax but is incompletely present in human populations in endemic regions. ${ }^{1-3}$ The presence of symptomatic or asymptomatic malaria can be used as an indicator of host immunity. Asymptomatic patients, through repeated infections, are more likely to have transmission-blocking antibodies like those against Pvs25 and Pvs28 that have been shown to reduce infectivity to mosquitoes. ${ }^{26}$ Indeed, asymptomatic $P$. vivax-infected individuals are less efficient transmitters, with only $13.5 \%$ patients infecting $0.45 \%$ of the mosquitoes with an average of 1.44 oocysts $( \pm 0.18) .{ }^{27}$ Similar results were reported by Alves et al., where the infection rate in mosquitoes was $1.2 \%$ for asymptomatic carriers versus $22 \%$ for symptomatic patients. $^{25}$ In symptomatic patients, other serum factors may modify the ability of $P$. vivax gametocytes to infect mosquitoes, as seen by enhanced mosquito infection rates after replacement of patient plasma with $P$. vivax-naïve sera or plasma. ${ }^{24}$ Future studies will compare mosquito infectivity of asymptomatic patients to that of symptomatic patients in association with determination of transmission-blocking antibodies and pro-inflammatory mediators.

It is possible that different genetic types of $P$. vivax could have contributed to variability in transmission. $P$. vivax has been classified into two types, VK210 and VK247, based on the differences in the Csp gene that encodes for the circumsporozoite protein, the major sporozoite surface protein. Both of these strains are equally prevalent in Peru, as shown by an equal number of pools of $A n$. darlingi being positive for VK210 and VK247. ${ }^{14}$ It will be of interest to investigate possible relationships of VK or other genetic types of parasite to transmission.

The presence or absence of gametocytes and their numbers might be considered a good predictor of mosquito infection by malaria parasites. Our study shows that the presence of gametocytes was higher in $P$. vivax transmitters than nontransmitters ( $94 \%$ vs. $72 \%$ ), and there was a strong correlation between gametocytemia and oocysts/midgut. This observation stands in contrast to an earlier study from Thailand that showed little relation between gametocyte density and either oocyst number or frequency of mosquito infection in $P$. vivax malaria. ${ }^{7}$ Unlike $P$. falciparum, the pyrogenic density of $P$. vivax is significantly lower and patients present earlier for medical care. Therefore, it may be possible that the peak gametocyte levels have not yet been achieved and there may be a relationship between the duration of symptoms and gametocytemia. Patients presenting later in the course of the disease may be contributing more to the continued transmission of $P$. vivax.

Gametocytemia as determined by real-time reverse transcriptase PCR correlated well with smear results. However, microscopic smear examination is quite variable and dependent on operator experience, so one might presume that realtime reverse transcriptase PCR might be more precise in measuring gametocytemia. Nonetheless, there was no correlation between molecular quantification of gametocytes and transmission of infection to mosquitoes, as determined by number of oocysts/midgut. Possible explanations for this might be (1) maturity of gametocytes (i.e., P. vivax gametocyte maturity 
might be heterogeneous, which would give a variable number of Pvs25 mRNAs per cell), (2) gametocyte sex ratio that might vary between strains, and (3) host response mechanisms that could alter gametocyte infectivity, such as antigametocyte antibodies, anemia, cytokines, nitric oxide, and chitotriosidase levels. Pro-inflammatory cytokines (TNF- $\alpha$, IL-1, and IL-6) and nitric oxide might influence the transmission of infection to mosquitoes by affecting gametocyte levels or their patency. At physiologic concentrations, recombinant $\mathrm{TNF}-\alpha$ is anti-parasitic, synergizing with IFN- $\gamma$ to induce production of nitric oxide and other toxic radicals. ${ }^{28}$ In the mouse model, C57BL/6 IFN- $\gamma^{-1-}$ mice infected with $P$. chabaudi AS produce lower amounts of other pro-inflammatory mediators, including $\mathrm{TNF}-\alpha$ and NO, with significantly higher parasitemia and mortality in comparison with wild-type controls. ${ }^{29}$ Nitric oxide has been shown to be cytostatic as well as cytotoxic to $P$. falciparum, $P$. berghei, and $P$. chabaudi in vitro. ${ }^{30}$ The infectivity of gametocytes, as shown by direct feeding of Anopheles stephensi mosquitoes on mice infected with $P$. vinckei petteri, has also been experimentally observed to be reduced during periods of schizogony that was reversed by injecting the L-arginine analogue, $N^{\omega}$-nitro-L-arginine (NwNLA), showing that the altered infectivity was due to reactive nitrogen intermediates (RNI). ${ }^{31}$ Although our study did not measure cytokine or NO levels, transmitters had lower temperature (although not statistically significant) and had significantly lower pulse rates, possible indicators of lower inflammatory mediators (IL- $1, \mathrm{TNF}-\alpha$ ), resulting in less damage to gametocytes with resulting higher level of transmission. Further studies are required to confirm this observation and determine the mechanistic relationship of proinflammatory mediators and parasite transmission.

The membrane feeding method to test transmissibility of parasites from humans to mosquitoes has been widely used with blood collected from both $P$. falciparum and $P$. vivax infected patients. ${ }^{32-37}$ When compared with direct feeds, membrane feeds have been found in Thailand to result in significantly fewer membrane feeds being infectious (53\% vs. $73 \%$ ). In one study, fewer mosquitoes were infected (50\% vs. $57 \%$ ) and fewer oocysts were produced per mosquito (8.8 vs. 13.4). ${ }^{24}$ Possible explanations for this difference are the artificial environment for mosquito feeding, effect of temperature change on gametocyte viability, the effect of handling blood on infectivity, and the use of colonized (used in Thailand) versus outbred mosquitoes (used in the present study). In situations where both experimental and control groups have used the membrane feeding method, this difference in transmission did not affect the final results. We used the membrane feeding method because it is preferred by volunteers and by international institutional review boards, and it has the additional advantage of allowing for experimental manipulation of patient blood. Importantly, we have shown here that $P$. vivax infection can be successfully transmitted to an outbred group of $A n$. darlingi mosquitoes. Most important, even in an area of low malaria transmission intensity, there appears to be a high level of transmission-blocking activity.

Transmission-blocking vaccines (TBV) work by inducing antibodies against the sexual stages (gamete, zygote, and ookinete) of the parasite that, when ingested along with a blood meal, block parasite development in the mosquito. Unlike vaccines against pre-erythrocytic and erythrocytic stages, TBVs will not prevent disease but are designed to reduce malaria transmission. Pvs25, expressed on the ookinete surface, is one such vaccine candidate that is undergoing clinical trial. ${ }^{38} \mathrm{~A}$ major emphasis in ongoing studies of transmissionblocking vaccines is to identify a developmentally regulated protein that induces transmission-blocking antibodies boosted by natural infection.

Measuring human infectivity and elucidating factors affecting it is the first step toward identifying potential transmission-blocking interventions that will complement currently available means. Knowing baseline transmission patterns of human-derived parasite infectivity for natural vectors in the field setting will provide the basis for determining the efficacy of transmission-blocking vaccines.

Received March 17, 2006. Accepted for publication June 15, 2006.

Acknowledgments: We are grateful to the Director of the Loreto Department of Health, Carlos Vidal Ore, M.D., for his support on this project. We thank our field team, Dr. Eddy Segura, Sonia Torres Andrade, and Nahir Chuquipiodo, along with the dedicated insectary staff, Victor Lopez, Carlos Tong, and Dario Ramirez; technical assistance in Iquitos from Carlos Pacheco, Flor Pacheco, Silvia Marin Gonzales, and Alex Tenorio Sanchez; helpful guidance from Kailash Patra and Mike Matthias; and participation of patients from the city of Iquitos and the villages of Varillal and Santo Tomas are also appreciated.

Financial support: This study was supported by an ASTMH-Ellison Postdoctoral Fellowship in Tropical Medicine (A.R.B.), NIH institutional training grant T32AI007036 (with which A.R.B. was supported), an IDSA summer scholarship (J.L.), the Baylor Department of International Medicine (J.L.), a Doris Duke Charitable Foundation Innovations in Clinical Research Program grant (J.M.V.), NIH/ NIAID grant K24AI068903 (J.M.V.), NIH/NIDA grant K01DA020364 (K.B.), and NIH Fogarty International Center Global Infectious Diseases Training Grant 5D43TW007120 (J.M.V.).

Authors' addresses: Ajay R. Bharti, Kimberly C. Brouwer, Jessica Lin, and Joseph M. Vinetz, University of California San Diego School of Medicine, La Jolla, California, E-mails: abharti@ucsd.edu, kbrouwer@ucsd.edu, jesstlin@gmail.com, and jvinetz@ucsd.edu. Raul Chuquiyauri and Alejandro Llanos-Cuentas, Instituto de Medicina Tropical Alexander von Humboldt, Universidad Peruana Cayetano Heredia, Lima, Peru, E-mails: raulharo@yahoo.com and allanos@upch.edu.pe. Jeffrey Stancil, Naval Medical Research Center Detachment, Iquitos, Peru, E-mail: stancil@nmrcd.med.navy.mil.

\section{REFERENCES}

1. Mendis KN, Munesinghe YD, de Silva YN, Keragalla I, Carter R, 1987. Malaria transmission-blocking immunity induced by natural infections of Plasmodium vivax in humans. Infect Immun 55: 369-372.

2. Munesinghe YD, Mendis KN, Carter R, 1986. Anti-gamete antibodies block transmission of human vivax malaria to mosquitoes. Parasite Immunol 8: 231-238.

3. Mendis KN, David PH, Carter R, 1990. Human immune responses against sexual stages of malaria parasites: considerations for malaria vaccines. Int J Parasitol 20: 497-502.

4. Roeffen W, Geeraedts F, Eling W, Beckers P, Wizel B, Kumar N, Lensen T, Sauerwein R, 1995. Transmission blockade of Plasmodium falciparum malaria by anti-Pfs230-specific antibodies is isotype dependent. Infect Immun 63: 467-471.

5. Healer J, McGuinness D, Hopcroft P, Haley S, Carter R, Riley E, 1997. Complement-mediated lysis of Plasmodium falciparum gametes by malaria-immune human sera is associated with antibodies to the gamete surface antigen Pfs230. Infect Immun 65: 3017-3023.

6. Lensen AH, Bolmer-Van de Vegte M, van Gemert GJ, Eling WM, Sauerwein RW, 1997. Leukocytes in a Plasmodium falciparum-infected blood meal reduce transmission of malaria to Anopheles mosquitoes. Infect Immun 65: 3834-3837.

7. Sattabongkot J, Maneechai N, Rosenberg R, 1991. Plasmodium 
vivax: gametocyte infectivity of naturally infected Thai adults. Parasitology 102: 27-31.

8. Mendis K, Sina BJ, Marchesini P, Carter R, 2001. The neglected burden of Plasmodium vivax malaria. Am J Trop Med Hyg 64: 97-106.

9. Roberts DR, Laughlin LL, Hsheih P, Legters LJ, 1997. DDT, global strategies, and a malaria control crisis in South America. Emerg Infect Dis 3: 295-302.

10. Nosten F, McGready R, Simpson JA, Thwai KL, Balkan S, Cho T, Hkirijaroen L, Looareesuwan S, White NJ, 1999. Effects of Plasmodium vivax malaria in pregnancy. Lancet 354: 546-549.

11. Vittor AY, Gilman RH, Tielsch J, Glass G, Shields T, Lozano WS, Pinedo-Cancino V, Patz JA, 2006. The effect of deforestation on the human-biting rate of Anopheles darlingi, the primary vector of falciparum malaria in the Peruvian Amazon. Am J Trop Med Hyg 74: 3-11.

12. Aramburu Guarda J, Ramal Asayag C, Witzig R, 1999. Malaria reemergence in the Peruvian Amazon region. Emerg Infect Dis 5: 209-215.

13. Schoeler GB, Flores-Mendoza C, Fernandez R, Davila JR, Zyzak M, 2003. Geographical distribution of Anopheles darlingi in the Amazon Basin region of Peru. J Am Mosq Control Assoc 19: 286-296.

14. Flores-Mendoza C, Fernandez R, Escobedo-Vargas KS, VelaPerez Q, Schoeler GB, 2004. Natural Plasmodium infections in Anopheles darlingi and Anopheles benarrochi (Diptera: Culicidae) from eastern Peru. J Med Entomol 41: 489-494.

15. Bottius E, Guanzirolli A, Trape JF, Rogier C, Konate L, Druilhe P, 1996. Malaria: even more chronic in nature than previously thought; evidence for subpatent parasitaemia detectable by the polymerase chain reaction. Trans R Soc Trop Med Hyg 90: 15-19.

16. Camargo EP, Alves F, Pereira da Silva LH, 1999. Symptomless Plasmodium vivax infections in native Amazonians. Lancet 353: $1415-1416$.

17. Camargo LM, Noronha E, Salcedo JM, Dutra AP, Krieger H, Pereira da Silva LH, Camargo EP, 1999. The epidemiology of malaria in Rondonia (Western Amazon region, Brazil): study of a riverine population. Acta Trop 72: 1-11.

18. Alves FP, Durlacher RR, Menezes MJ, Krieger H, Silva LH, Camargo EP, 2002. High prevalence of asymptomatic Plasmodium vivax and Plasmodium falciparum infections in native Amazonian populations. Am J Trop Med Hyg 66: 641-648.

19. Roshanravan B, Kari E, Gilman RH, Cabrera L, Lee E, Metcalfe J, Calderon M, Lescano AG, Montenegro SH, Calampa C, Vinetz JM, 2003. Endemic malaria in the Peruvian Amazon region of Iquitos. Am J Trop Med Hyg 69: 45-52.

20. Branch O, Casapia WM, Gamboa DV, Hernandez JN, Alava FF, Roncal N, Alvarez E, Perez EJ, Gotuzzo E, 2005. Clustered local transmission and asymptomatic Plasmodium falciparum and Plasmodium vivax malaria infections in a recently emerged, hypoendemic Peruvian Amazon community. Malar J 4: 27.

21. Marrelli MT, Honorio NA, Flores-Mendoza C, Lourenco-deOliveira R, Marinotti O, Kloetzel JK, 1999. Comparative susceptibility of two members of the Anopheles oswaldoi complex, An. oswaldoi and An. konderi, to infection by Plasmodium vivax. Trans $R$ Soc Trop Med Hyg 93: 381-384.

22. Drakeley CJ, Jawara M, Targett GA, Walraven G, Obisike U, Coleman R, Pinder M, Sutherland CJ, 2004. Addition of artesunate to chloroquine for treatment of Plasmodium falciparum malaria in Gambian children causes a significant but shortlived reduction in infectiousness for mosquitoes. Trop Med Int Health 9: 53-61.

23. Tsuboi T, Kaslow DC, Gozar MM, Tachibana M, Cao YM, Torii M, 1998. Sequence polymorphism in two novel Plasmodium vivax ookinete surface proteins, Pvs 25 and Pvs28, that are malaria transmission-blocking vaccine candidates. Mol Med 4: $772-782$.
24. Sattabongkot J, Maneechai N, Phunkitchar V, Eikarat N, Khuntirat B, Sirichaisinthop J, Burge R, Coleman RE, 2003. Comparison of artificial membrane feeding with direct skin feeding to estimate the infectiousness of Plasmodium vivax gametocyte carriers to mosquitoes. Am J Trop Med Hyg 69: 529-535.

25. Alves FP, Gil LH, Marrelli MT, Ribolla PE, Camargo EP, Da Silva LH, 2005. Asymptomatic carriers of Plasmodium spp. as infection source for malaria vector mosquitoes in the Brazilian Amazon. J Med Entomol 42: 777-779.

26. Sattabongkot J, Tsuboi T, Hisaeda H, Tachibana M, Suwanabun N, Rungruang T, Cao YM, Stowers AW, Sirichaisinthop J, Coleman RE, Torii M, 2003. Blocking of transmission to mosquitoes by antibody to Plasmodium vivax malaria vaccine candidates Pvs 25 and Pvs28 despite antigenic polymorphism in field isolates. Am J Trop Med Hyg 69: 536-541.

27. Coleman RE, Kumpitak C, Ponlawat A, Maneechai N, Phunkitchar V, Rachapaew N, Zollner G, Sattabongkot J, 2004. Infectivity of asymptomatic Plasmodium-infected human populations to Anopheles dirus mosquitoes in western Thailand. $J$ Med Entomol 41: 201-208.

28. Anstey NM, Weinberg JB, Hassanali MY, Mwaikambo ED, Manyenga D, Misukonis MA, Arnelle DR, Hollis D, McDonald MI, Granger DL, 1996. Nitric oxide in Tanzanian children with malaria: inverse relationship between malaria severity and nitric oxide production/nitric oxide synthase type 2 expression. J Exp Med 184: 557-567.

29. Su Z, Stevenson MM, 2000. Central role of endogenous gamma interferon in protective immunity against blood-stage Plasmodium chabaudi AS infection. Infect Immun 68: 4399-4406.

30. Balmer P, Phillips HM, Maestre AE, McMonagle FA, Phillips RS, 2000. The effect of nitric oxide on the growth of Plasmodium falciparum, P. chabaudi and P. berghei in vitro. Parasite Immunol 22: 97-106.

31. Motard A, Landau I, Nussler A, Grau G, Baccam D, Mazier D, Targett GA, 1993. The role of reactive nitrogen intermediates in modulation of gametocyte infectivity of rodent malaria parasites. Parasite Immunol 15: 21-26.

32. Graves PM, Burkot TR, Carter R, Cattani JA, Lagog M, Parker J, Brabin BJ, Gibson FD, Bradley DJ, Alpers MP, 1988. Measurement of malarial infectivity of human populations to mosquitoes in the Madang area, Papua, New Guinea. Parasitology 96: $251-263$.

33. Ramsey JM, Salinas E, Rodriguez MH, Beaudoin RL, 1994. Effects of transmission-blocking immunity on Plasmodium vivax infections in Anopheles albimanus populations. J Parasitol 80: $88-92$.

34. Gamage-Mendis AC, Rajakaruna J, Carter R, Mendis KN, 1992. Transmission blocking immunity to human Plasmodium vivax malaria in an endemic population in Kataragama, Sri Lanka. Parasite Immunol 14: 385-396.

35. Somboon P, Suwonkerd W, Lines JD, 1994. Susceptibility of Thai zoophilic Anophelines and suspected malaria vectors to local strains of human malaria parasites. Southeast Asian J Trop Med Public Health 25: 766-770.

36. Ramsey JM, Salinas E, Rodriguez MH, 1996. Acquired transmission-blocking immunity to Plasmodium vivax in a population of southern coastal Mexico. Am J Trop Med Hyg 54: 458-463.

37. Chan AS, Rodriguez MH, Torres JA, Rodriguez MC, Villarreal C, 1994. Susceptibility of three laboratory strains of Anopheles albimanus (Diptera: Culicidae) to coindigenous Plasmodium vivax in southern Mexico. J Med Entomol 31: 400-403.

38. Malkin EM, Diemert DJ, McArthur JH, Perreault JR, Miles AP, Giersing BK, Mullen GE, Orcutt A, Muratova O, Awkal M, Zhou H, Wang J, Stowers A, Long CA, Mahanty S, Miller LH, Saul A, Durbin AP, 2005. Phase 1 clinical trial of apical membrane antigen 1: an asexual blood-stage vaccine for Plasmodium falciparum malaria. Infect Immun 73: 3677-3685. 